\title{
Energy transfer from retinal to amino acids - a time-resolved study of the ultraviolet emission of bacteriorhodopsin
}

\author{
H.J. Polland ${ }^{a}$, M.A. Franz ${ }^{\text {a }}$, W. Zinth ${ }^{a}$, W. Kaiser ${ }^{a}$ and D. Oesterhelt ${ }^{b}$ \\ a Physik Department der Technischen Universität München, Munich, \\ and ${ }^{b}$ Max-Planck-Institut für Biochemie, D-8033 Martinsried (F, R.G.)
}

(Received 7 March 1986)

Key words: Energy transfer; Retinal; Bacteriorhodopsin

Two-step excitation of retinal in bacteriorhodopsin by visible light is followed by an energy transfer to amino acids that is seen as fluorescent emission around $350 \mathrm{~nm}$. The fluorescence spectrum obtained after two-step excitation $(2 \times 527 \mathrm{~nm})$ differs from the fluorescence spectrum obtained after one-step ultraviolet excitation (263.5 $\mathrm{nm}$ ) by a strongly quenched emission with a fluorescence lifetime of $10 \pm 5 \mathrm{ps}$ and a smaller spectral width. The two-step absorption process presumably selects tryptophan residues which strongly couple to the retinal chromophore.

\section{Introduction}

Many proteins contain, besides the amino acids, other functionally important molecules, prosthetic groups. Sometimes these molecules are coloured compounds, e.g., porphyrins, carotenoids and retinals that absorb light in the visible spectral region. The spatial arrangement of these dye molecules and the amino acid side chains of the protein which form the binding site is of fundamental interest for understanding the function of the proteins, for example the function of halobacterial proteins as light-energy converters or sensors. Even though the precise structure of the binding sites can only be established by X-ray crystallographic analysis of the entire chromoprotein, spectroscopic techniques, e.g., nuclear magnetic resonance (NMR) or optical spectroscopy can be used to identify amino acid side chains that interact with the dye molecules. Here we describe the spatial connection between retinal and the sur-

Correspondence address: Dr. D. Oesterhelt, Max-Planck-Institut für Biochemie, D-8033 Martinsried, F.R.G. rounding amino acids in bacteriorhodopsin as analysed by picosecond time-resolved optical methods.

\section{General considerations}

Amino acids absorb light with wavelengths shorter than $200 \mathrm{~nm}$ and only the aromatic amino acids phenylalanine, tyrosine and tryptophan have red-shifted absorption bands around $280 \mathrm{~nm}$ [1]. Even in small proteins it is impossible to make unique assignments of absorption or fluorescence bands to specific amino acid residues in the protein because the spectral bands are rather broad. The situation is more complex and at the same time more informative, when a dye molecule is incorporated into the protein, for example retinal into bacterioopsin: two groups of amino acids in the protein can now be distinguished (Fig. 1). A first group (I) consists of all amino acids that do not couple directly to the exited levels of the dye molecule. These amino acids show broad absorptions and fluorescence lifetimes $\tau_{\mathrm{f}} \approx 10^{-9}$ s. A second group (II) consists of a few residues that couple excitonically (strongly) to higher lying elec- 


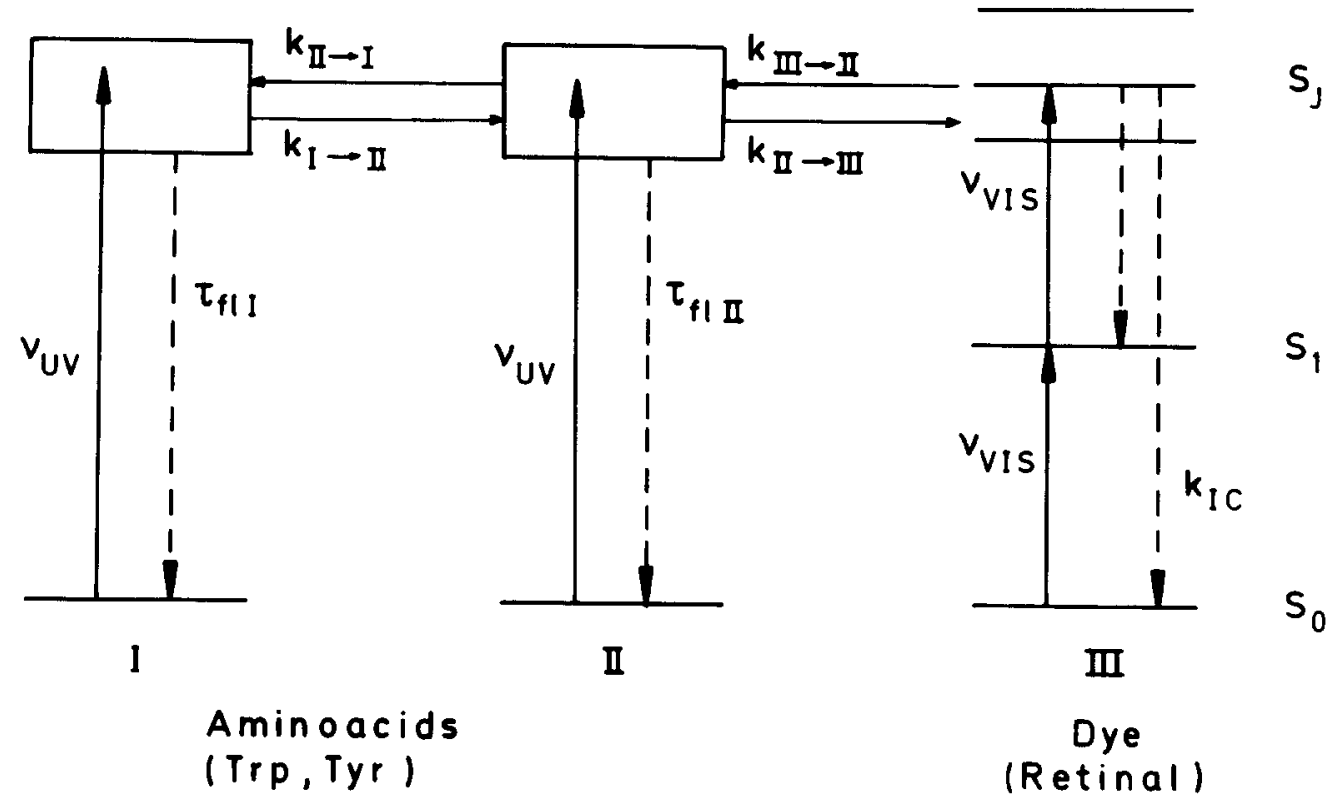

Fig. 1. Scheme of the energy levels which determine the ultraviolet fluorescence in biological systems, when a dye molecule (III) is incorporated into the protein. Amino acids weakly coupled to the dye molecule are type I, amino acids strongly coupled to the dye molecule are type II. The properties of the ultraviolet fluorescence depend strongly on energy transfer and relaxation rates $k$.

tronic levels $\left(S_{J}\right.$ with $\left.J>1\right)$ of the dye molecules (III). It is known for many dye molecules that an excitation of the electronic states $S$, with $J>1$ is transferred very rapidly into the lowest level $S_{1}$ with time constants less than 1 ps [2]. The strong coupling of the amino acids from group II to the short-lived level $S_{J}$ with $J>1$ of the dye molecules causes an effective quenching of the fluorescence of the respective amino acids after excitation.

Previous reports have suggested that tryptophan residues participate in the chromophoric structure of bacteriorhodopsin [3,4]. Energy transfer from aromatic residues to retinol in the binding site depends on the stereochemistry of the retinyl moiety, the all-trans and the 13-cis configuration being the most effective ones [5]. Energy transfer from aromatic amino acid residues to the retinylidene moiety in native bacteriorhodopsin was shown by fluorescence quenching and photochemical studies. Data indicated that tyrosine residues were not likely to be responsible for these effects [6]. However, further structural or quantitative conclusions are difficult because amino acids of group I and group II are always excited simultaneously and the fluorescence of both groups overlaps yielding only unspecific information. More details are obtained, when the amino acids of group II are excited exclusively. This is accomplished for bacteriorhodopsin in the following way: excitation of the state $S_{J}$ of the retinal (dye) will lead (due to the excitonic coupling between retinal and the amino acids) to a certain excitation of the amino acids of group II, which is reflected by a fluorescence emission from these amino acids. Unfortunately, the strong and broad ultraviolet absorption of all aromatic amino acids fall together with the ultraviolet absorption band of retinal in bacteriorhodopsin preventing its selective excitation via ultraviolet light. On the other hand, the excitation of retinal with visible light may serve to populate the levels $S_{J}$ by a two-step excitation process. Starting from the ground state $S_{0}$ of the dye molecule a first exciting pulse populates the level $S_{1}$. Within in the lifetime of the state $S_{1}$ a second light pulse can promote the dye molecules to the level $S_{J}$ from which the amino acids of group II are populated. The quantities one can 
measure are the following. (1) Fluorescence emission spectra after ultraviolet excitation and quenching of aromatic amino acid fluorescence upon formation of the chromophore by retinal addition give information on the bulk properties of group I and II. (2) Ultraviolet fluorescence emission spectra after two-step excitation by visible light give information exclusively on amino acids of group II. (3) Time-dependent measurements of the fluorescence emissions after ultraviolet and two-step visible excitation show the strength of the excitonic coupling between the amino acids and the dye molecules. The experiments described in this paper apply the above ideas to the system of bacteriorhodopsin (for a recent review, see Ref. 7).

\section{Materials and Methods}

Purple membrane was prepared as described in Ref. 8 and the retinal removed by treatment with hydroxylamine and light as in Ref. 9. The apomembrane (bacterioopsin) obtained was either reconstituted with 9,12-phenylretinal [10] or with all-trans and 13-cis retinal purified by high-pressure liquid chromatography. Reconstitution of the chromoproteins was followed spectroscopically in an Aminco DW2 spectrophotometer [11] and fluorescence emission spectra ( $\lambda$ excitation $280 \mathrm{~nm}$ ) recorded in a Perkin Elmer $\mathrm{MPF}_{3}$ spectrofluorimeter. Except in the experiment of Fig. 3 suspensions with an optical density of $1-1.5$ at $\lambda=570 \mathrm{~nm}$ for bacteriorhodopsin and $\lambda=490 \mathrm{~nm}$ for 9,12-phenylbacteriorhodopsin were used. The optical path length was $1 \mathrm{~mm}$, thus giving a minimal transmission $T_{\min } \approx 50 \%$ in the spectral region between $\lambda=300 \mathrm{~nm}$ and $\lambda=700 \mathrm{~nm}$ and minimizing reabsorption of fluorescence light. Prior to each experiment the sample was light-adapted for $30 \mathrm{~min}$ $(150 \mathrm{~W}, \lambda \geqslant 400 \mathrm{~nm})$.

The experiments with ultrafast excitation pulses were performed with single picosecond pulses $\left(t_{\mathrm{p}}\right.$ $=4 \mathrm{ps)}$ of low repetition rate from a mode-locked $\mathrm{Nd}$ : glass laser system. Fluorescence of the sample was recorded after both, one-step (ultraviolet excitation with $\lambda=263.5 \mathrm{~nm}$ from the fourth harmonic of the $\mathrm{Nd}$ : glass laser) and two-step excitation (visible excitation (visible excitation in the absorption band of bacteriorhodopsin by $\lambda=527 \mathrm{~nm}$ from the second harmonic of the $\mathrm{Nd}$ : glass laser). We note that the total energy applied to each bacteriorhodopsin molecule is the same for the two excitation processes. In order to avoid saturation processes only low irradiance was applied. The peak intensity of the pulses was less than 300 $\mathrm{MW} / \mathrm{cm}^{2}$. During one excitation process less than $15 \%$ of the bacteriorhodopsin molecules in the beam absorbed one photon.

For the time-resolved measurement, the fluorescence emission between $\lambda=300 \mathrm{~nm}$ and $\lambda=$ $400 \mathrm{~nm}$ was registered with a streak camera. The streak curves were corrected for non-linearities in the detection system. The time resolution of the experimental system was determined by the sweep speed of the streak camera to 10 ps [16].

The fluorescence spectra were resolved with a $25 \mathrm{~cm}$ grating spectrometer (spectral resolution $\Delta \lambda=5 \mathrm{~nm}$ ) and an ultraviolet-sensitive photomultiplier. The signal was recorded in the forward direction. All spectra shown are corrected for the spectral response of the detection system and for re-absorption of the sample. Absorption spectra of the bacteriorhodopsin samples were recorded prior to and after each experiment $(250 \mathrm{~nm} \leqslant \lambda \leqslant 700$ $\mathrm{nm})$. No difference in absorption indicative of denaturation of the material during the experiments was observed.

\section{Results}

\section{The fluorescence spectra}

Illuminating bacteriorhodopsin by ultraviolet picosecond pulses at $\lambda=263.5 \mathrm{~nm}$ or by visible pulses at $\lambda=527 \mathrm{~nm}$ always resulted in a distinct fluorescence emission between $280 \mathrm{~nm}$ and 400 nm. Two fluorescence spectra are presented in Fig. 2. The first of the fluorescence spectra is obtained at the excitation wavelength $\lambda=263.5$ $\mathrm{nm}$ in the ultraviolet (Fig. 2 - - - $\mathbf{0}$ ). The total fluorescence intensity in this experiment depends linearly on the excitation energy (as is typical for a one-photon process). A broad emission extends from $300 \mathrm{~nm}$ to $400 \mathrm{~nm}$ with the peak of emission at $\lambda=315 \pm 5 \mathrm{~nm}$. The fluorescence spectrum has a halfwidth $\Delta \lambda=55 \mathrm{~nm}$. It is very similar to previously reported fluorescence spectra [12]. It should be noted that the same fluorescence spec- 


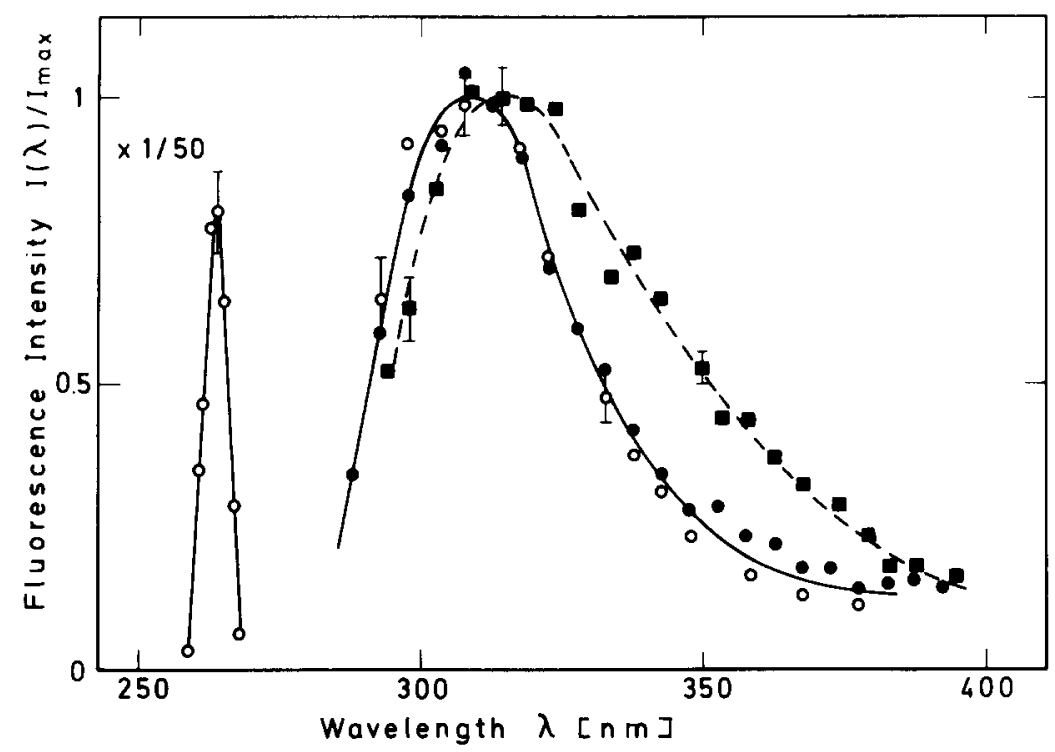

Fig. 2. Emission spectra of bacteriorhodopsin recorded in the ultraviolet spectral range after resonant excitation with ultraviolet light ( $\lambda=263.5 \mathrm{~nm}$, dashed curve, squares) and two-step visible excitation ( $\lambda=527 \mathrm{~nm}$, solid curve, open circles). Measurements using the modified 9,12-phenylbacteriorhodopsin gave very similar two-step excitation fluorescence (full circles).

trum is obtained with both picosecond flash and continuous excitation.

A somewhat different spectrum (Fig. 2 $\mathrm{O}-\mathrm{O}$ ) is found when excitation occurs via picosecond pulses at $\lambda=527 \mathrm{~nm}$. The resulting emission consists of two different bands: (i) a broad band between $\lambda=280 \mathrm{~nm}$ and $\lambda=400 \mathrm{~nm}$ as seen before; and (ii) a narrow band at $\lambda=263.5$ $\mathrm{nm}$. Compared with ultraviolet excitation details of the broad fluorescence band are modified: the maximum emission occurs at $\lambda=305 \pm 5 \mathrm{~nm}$ instead at $\lambda_{\max }=315 \mathrm{~nm}$ and the spectral halfwidth is smaller with $\Delta \lambda=40 \mathrm{~nm}$. The spectrally integrated intensity increases with the square of the excitation intensity as is expected for two-photon or two-step excitation processes. At an excitation level of 0.1 absorbed photons per bacteriorhodopsin molecule we estimate a quantum yield of $\eta=2 \cdot 10^{-5}$ for the two-step absorption/ fluorescence process. This quantum yield is about 1000-times smaller than that of the one-step ultraviolet excitation [7]. The second emission band in the two-step excitation experiment is observed at $\lambda=263.5 \mathrm{~nm}$ which is exactly the second harmonic of the excitation wavelength. The linewidth of this band is smaller than the resolution of our spec- trometer, i.e., smaller than $\Delta \lambda=5 \mathrm{~nm}$. This emission is related to the ordered arrangement of the bacteriorhodopsin molecules in the purple membrane because bacteriorhodopsin preparations after treatment with octylglucoside which destroys the two-dimensional hexagonal lattice of the purple membrane do not show any longer this emission [13]. This finding demonstrates that the feature at $263.5 \mathrm{~nm}$ is not due to a real photobiological process, but is caused by a nonlinear optical phenomenon, the so-called second harmonic generation. Because second harmonic generation is only possible in media that do not have inversion symmetry on the distance scale of one wavelength [14], the second harmonic peak is only present in purple membranes but is absent in monomeric bacteriorhodopsin samples.

The experiments with the excitation at $\lambda=527$ $\mathrm{nm}$ were repeated with two additional samples. First, retinal was extracted from the purple membrane and by doing this a chromophore-free suspension of bacterioopsin prepared [10]. Exciting this sample with light at $527 \mathrm{~nm}$ at the same intensity as used above did not give an ultraviolet emission within the dynamic range of our experiment. Second, a chromoprotein was reconstituted 
by addition of the sterically hindered 9,12-phenylretinal. This retinal analogue forms a chromoprotein (9,12-phenylbacteriorhodopsin) with an absorption maximum ( $\left.\lambda_{\max } 490 \mathrm{~nm}\right)$ considerably different from bacteriorhodopsin $\left(\lambda_{\max } 570\right)$ and is photochemically inactive [11]. The emission spectrum of 9,12-phenylbacteriorhodopsin upon two-step excitation is compared (Fig. 2 - - - ) with that of bacteriorhodopsin $\left(\mathrm{O}_{-}-\mathrm{O}\right)$ in Fig. 2. Maximum emission of 9,12-phenylbacteriorhodopsin occurs at $\lambda=305 \mathrm{~nm}$ with a narrow spectral halfwidth of $\Delta \lambda=40 \mathrm{~nm}$, i.e., no difference can be found between the ultraviolet emission spectra of bacteriorhodopsin and 9,12-phenylbacteriorhodopsin in spite of the different absorption properties in the visible $(\Delta \lambda=80 \mathrm{~nm})$.

The experiment with 9,12-phenylbacteriorhodopsin provides a good control to exclude the possibility of fluorescence emission from photoproducts formed in bacteriorhodopsin during the 4 ps excitation period ( $J$ : rise time, $430 \mathrm{fs}, K$ : rise time, 5 ps). In 9,12-phenylbacteriorhodopsin the only populated state is $S_{1}$ that decays with a half-time of 10 ps directly to the ground state and no photoproducts are formed [11].
To quantify the amino acids of groups I and II, the fluorescence quenching upon association of retinal and bacterioopsin was used. Fig. 3 shows the results of such an experiment. Bacteriorhodopsin was reconstituted from bacterioopsin by stepwise addition of 13-cis retinal and the course of the reaction followed by measuring the absorbance change at $570 \mathrm{~nm}$ (Fig. 3a) and by measuring the fluorescence emission change at 315 $\mathrm{nm}$ upon excitation at $280 \mathrm{~nm}$ (Fig. 3b). Both changes correlate. A linear increase in absorption corresponds to a linear decrease in fluorescence. This quenching of aromatic amino acid fluorescence ends at a molar ratio of retinal-tobacterioopsin of $1: 1$. Extrapolation allows one to estimate that about $2 / 3$ of the original ultraviolet fluorescence are quenched by retinal (see Discussion). No apparent difference in the results was found when all-trans retinal instead of 13-cis retinal was used at a temperature of $4-6^{\circ} \mathrm{C}$, where the respective isomers require hours to equilibrate thermally, thus indicating a large similarity of 13-cis retinal and all-trans retinal with respect to their interaction with aromatic amino acid residues.

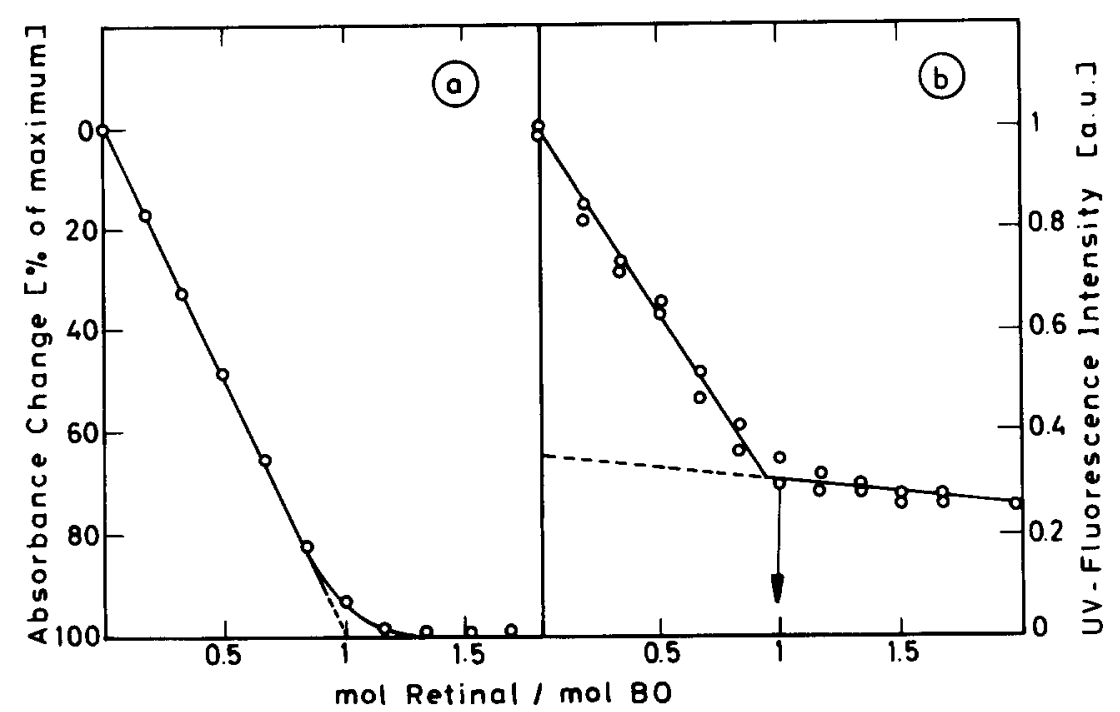

Fig. 3. Titration of bacteriopsin (BO) with 13-cis retinal. (a) Reconstitution of bacteriorhodopsin measured as increase in optical density at $570 \mathrm{~nm}$ and given in percent of the final value reached at a molar ratio of 1. Retinal was added as an isopropanolic solution $(1 \mathrm{mM})$ in microlitre aliquots to $2 \mathrm{ml}$ of an apomembrane suspension. (b) Ultraviolet fluorescence quenching upon chromophore formation. Fluorescence intensity in the samples from (a) was measured after excitation at $280 \mathrm{~nm}$ and readings taken in duplicate. In a control experiment it was shown that the isopropanol did not influence the fluorescence intensity. 
Reconstitution of bacteriorhodopsin in the purple membrane involves cooperativity of binding sites, recrystallization of the protein and conformational changes [17-19]. All three phenomena could also contribute to fluorescence quenching of tryptophan residues in bacteriorhodopsin. Ultraviolet fluorescence changes indeed have been observed during the transition from bacteriorhodop$\sin$ to its $M$ state [20], which shows a similar interaction between pigment and protein as free retinal and bacterioopsin [21]. These fluorescent changes are seen as a 5\% increase during reformation of bacteriorhodopsin compared to almost $70 \%$ quenching observed during reconstitution of bacteriorhodopsin [20]. We therefore conclude that the quenching must mainly arise from the coupling of retinal with aromatic amino acids.

\section{Time-resolved fluorescence measurements}

By means of a streak camera the time dependence of the ultraviolet fluorescence emission (wavelengths $300 \mathrm{~nm} \leqslant 400 \mathrm{~nm}$ ) after excitation with picosecond light pulses $\left(\lambda_{e x}=263.5 \mathrm{~nm}\right)$ could be measured. In a first experiment, the chromophore-free preparation of bacterioopsin was studied and a fluorescence decay time of 1.25 ns could be observed. The experimental data for the investigation of native bacteriorhodopsin containing the chromophore are shown in Fig. 4a. The fluorescence signal rises rapidly with the time resolution of the streak camera. Subsequently, it decays exponentially with a time constant of $\tau_{\mathrm{r}}=$ $550 \mathrm{ps}$, i.e., the fluorescence decay in bacteriorhodopsin is faster by a factor of 2 than the fluorescence decay in bacterioopsin. Within the dynamic range of our measurements we did not find a bi-exponential behaviour for neither of the samples, bacteriorhodopsin nor bacterioopsin (see Discussion). A completely different time dependence of the fluorescence emission is found when working with excitation pulses at $\lambda=527 \mathrm{~nm}$ (Fig. $4 \mathrm{~b})$. In this case the decay is accelerated by a factor of 50, i.e., a decay time $\tau_{\mathrm{r}}=10 \pm 5$ is found which is close to the time resolution of the experimental set up used. This short decay time is in agreement with the low fluorescence quantum yield of $2 \cdot 10^{-5}$ ps found in the two-step excitation process. In various additional experiments we ascertained that the recorded signal was caused by

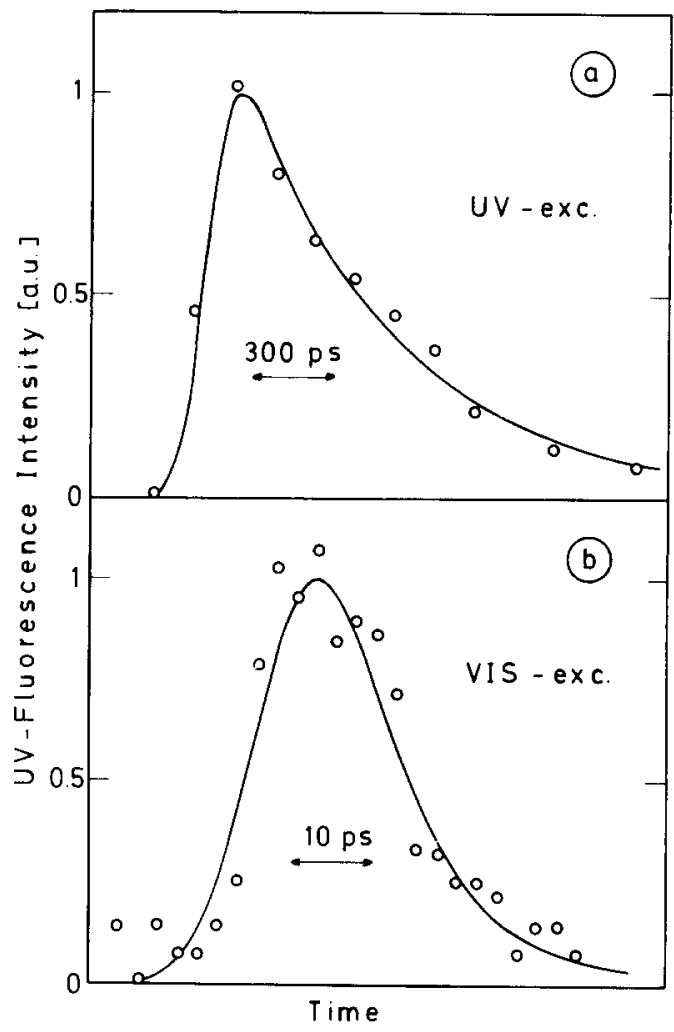

Fig. 4. Time dependence of the ultraviolet fluorescence emission: (a) after ultraviolet excitation, $\lambda=263.5 \mathrm{~nm}, t_{\mathrm{p}}=4 \mathrm{ps}$ (time resolution of the streak-camera system, $50 \mathrm{ps)}$ and (b) two-step visible excitation $\lambda=527 \mathrm{~nm}, t_{\mathrm{p}}=4 \mathrm{ps}$ (time resolution, $10 \mathrm{ps}$ ). Note the much faster decay of the ultraviolet fluorescence after the two-step visible excitation process.

bacteriorhodopsin itself and not by stray light from impurities of the samples.

\section{Discussion}

The experimental results allow one to discuss the path of the energy flow in bacteriorhodopsin after ultraviolet and after two-step excitation and provide information on the amino acids excitonically coupled to the retinal moiety in the chromophoric structure. Using the ultraviolet excitation wavelength of $263.5 \mathrm{~nm}$ mainly amino acids containing aromatic groups, such as tyrosine, tryptophan, and phenylalanine, are excited [15]. Only a small fraction of about $10 \%$ of the light will be absorbed in bacteriorhodopsin by the retinal molecule [22]. From all the amino acids only 
tyrosine and tryptophan are strongly fluorescent at wavelengths longer than $300 \mathrm{~nm}$, but tyrosine fluorescence was shown to be fully quenched in bacteriorhodopsin [6]. We therefore conclude that the emission observed in our experiments is caused mainly by tryptophan residues. The presence of the dye molecule retinal in bacteriorhodopsin leads to a reduction of the fluorescence lifetime of the amino acids from $1.25 \mathrm{~ns}$ in bacterioopsin to $\tau=550 \mathrm{ps}$ in bacteriorhodopsin.

At the same time fluorescence intensity decreases to about one-third. The average radiative $\left(\tau_{\text {rad }}\right)$ lifetime of the fluorescence can be calculated as shown below to be about $5.5 \mathrm{~ns}$ and allows an estimation of the percentage of the fluorescent tryptophan residues quenched in bacteriorhodopsin in the following way. Because the overall decay of fluorescence in bacterioopsin (BO) and bacteriorhodopsin (BR) follow single exponentials the fluorescent decay time $\left(\tau_{\mathrm{fl}}\right)$ of the individual tryptophans must be very similar and the values of $1.25 \mathrm{~ns}\left(\tau_{\mathrm{f}}^{\mathrm{BO}}\right)$ and about $500 \mathrm{ps}$ $\left(\tau_{\mathrm{fl}}^{\mathrm{BR}}\right)$ can be taken as average. The quantum yield then is given by

$\phi=\frac{\sum \phi_{i}}{N}=\frac{\tau_{\Pi}}{\tau_{\text {rad }}}$

where $N$ is the number of tryptophan residues in BO ( $N=8$, see Ref. 23 and $\phi_{i}$ is the fluorescence quantum yield of the individual residues in bacteriorhodopsin. We assume that in bacterioopsin the quantum efficiencies are equal, $\phi_{i}=\phi_{0}$ and that in bacteriorhodopsin $x$ residues are quenched, $\phi_{x}=0$. Then the number of fluorescence quenched residues in bacteriorhodopsin can be estimated by Eqn. 1.

$$
\begin{aligned}
& \frac{\tau_{f}^{\mathrm{BR}}}{\tau_{\mathrm{rad}}}=\frac{\sum_{\phi_{i}}}{N}=\frac{(N-x) \phi_{0}}{N}=\frac{N-x}{N} \frac{\tau_{\mathrm{f}}^{\mathrm{BO}}}{\tau_{\mathrm{rad}}} \\
& x=N\left(1-\frac{\tau_{f}^{\mathrm{BR}}}{\tau_{\mathrm{fi}}^{\mathrm{BO}}}\right)
\end{aligned}
$$

The resulting value of $x=4.8$ indeed correlates well with the observed quenching of two-thirds of all tryptophan residues and confirms conclusions drawn by Acuna et al. [24]. Considering the most likely structural models put forward for bacteriorhodopsin [25], the tryptophan residues
$80,86,182$ and 189 are the most likely candidates for interaction with bacteriorhodopsin whereas Trp 137, 138 (helix E) following each other in sequence on the $\alpha$ helix might less well interact and $\operatorname{Trp} 11$ and 12 are not located in the neighbourhood of retinal at all. Three groups reported on bi-exponential decay of bacterioopsin and bacteriorhodopsin $[24,26,6]$, assigning different amplitudes and half-times to the various components. Since the experimental conditions, such as solvents, emission maxima used for analysis, etc., were different, no comprehensive evaluation of the differences is possible.

The effective quenching of the ultraviolet fluorescence due to the retinal molecule indicates that excitonic coupling between the amino acids themselves and between amino acids and the retinal molecule exists. This finding is in agreement with data from the literature where a considerably large quantum efficiency for the photocycle of bacteriorhodopsin was found upon ultraviolet excitation of the amino acids [6]. Consequently, the exchange rates $k$ in the model of Fig. 1 are large, $k>10^{9}$ $\mathrm{s}^{-1}$, and the excitation is dissipated over amino acids of group II and also reaches the quenching chromophore. Excitation of bacteriorhodopsin via a two-step absorption process with visible light leads to ultraviolet fluorescence with properties differing from those found in the ultraviolet excitation experiments. The possibility that fluorescence from the excited electron state of the retinal molecule itself was observed can be ruled out because the fluorescence spectrum of a molecule must depend strongly on its energy level structure. Consequently, different spectra should be obtained when investigating different types of retinal molecules in the binding site. Retinal and the 9,12-phenylretinal considerably differ in structure and in the energies of the first excited singlet state $S_{1}$ [11]. On the other hand, when these molecules are incorporated into bacterioopsin we observe very similar ultraviolet fluorescence spectra after a two-step visible excitation. This is a strong indication that the observed ultraviolet fluorescence does not originate from the excited singlet state of retinal, but from strongly coupled amino acids.

The short fluorescence lifetime, the lack of a long-lived fluorescence emission and the low fluorescence quantum yield indicate that mainly one 
group of amino acids is strongly coupled to the retinal molecule and that the coupling of these amino acids to the other amino acids is much weaker, i.e., $K_{\mathrm{III} \rightarrow \mathrm{II}} \gg K_{\mathrm{II} \rightarrow \mathrm{I}}$ (Fig. 1).

The data indicate that the most strongly coupled amino acids are tryptophans (confirming the observation reported in Ref. 6). The measured quantum efficiency of $\eta=2 \cdot 10^{-5}$ upon visible excitation is used together with experimental parameters to derive a value for the radiative lifetime of the residues in question as follows:

$\eta=\frac{\eta_{\mathrm{III} \rightarrow \mathrm{II}} \tau_{\mathrm{SI}} \tau_{\mathrm{fI}} W}{t_{\mathrm{p}} \tau_{\mathrm{rad}}}$

$\eta_{\mathrm{III} \rightarrow \mathrm{II}}$ is the excitation-transfer probability between the excited electronic state $S_{1}$ of the retinal and the excited electronic state of the amino acid (type II) and should be close to 1 [6]. $\tau_{\mathrm{S} 1}=0.43 \mathrm{ps}$ is the lifetime of the $S_{1}$ state of bacteriorhodopsin [13]; $t_{\mathrm{p}}=4 \mathrm{ps}$ is the duration of the visible excitation pulse; $\tau_{\mathrm{f}}=10 \mathrm{ps}$ is the measured fluorescence lifetime; $W=0.1$ is the percentage of excited bacteriorhodopsin molecules in the first excitation step. We also assume that the absorption crosssection for the absorption of light at $527 \mathrm{~nm}$ from the ground state to the $S_{1}$ state of bacteriorhodopsin and from the $S_{1}$ state to the higher lying singlet state are the same [16]. $\tau_{\text {rad }}$ is the radiative lifetime of the fluorescence state. From Eqn. 4 we estimate the radiative lifetime $\tau_{\text {rad }}$ to be $\tau_{\text {rad }} \leqslant 5.5$ $\mathrm{ns}\left(\tau_{\mathrm{rad}}=5.5 \mathrm{~ns}\right.$ holds for the highest value of the excitation transfer $\left.\left(\eta_{\mathrm{III} \rightarrow \mathrm{II}}=1\right)\right)$.

An estimate of radiative lifetimes of the amino acids according to Ref. 15 leads to $50 \mathrm{~ns}$ for tyrosines and $13 \mathrm{~ns}$ for tryptophans. The radiative lifetime of tryptophan is within the employed approximations and the accuracy in agreement with the computed value of $5.5 \mathrm{~ns}$. We conclude that the amino acids fluorescing after visible twostep excitation are tryptophans.

In the literature it had been proposed that the ultraviolet fluorescence of bacteriorhodopsin after direct ultraviolet excitation is determined by two types of tryptophans which were in hydrophobic (shorter wavelengths) and hydrophilic (longer wavelengths) environments, respectively [8]. The narrowing of the ultraviolet emission and the shift of its peak to shorter wavelengths with two-step excitation indicate that tryptophan which couples to the retinal is in a hydrophobic surrounding.

\section{Conclusion}

In bacteriorhodopsin a strong excitonic coupling between the retinal chromophore and amino acids, presumably tryptophans, exists. The excitonic coupling is so efficient that the two-step excitation of the chromophore by visible light leads to a considerable ultraviolet fluorescence from the tryptophan molecules. The short lifetimes of higher excited electronic states of retinal of up to $10^{-12} \mathrm{~s}$ suggest that this excitation exchange is very rapid. The data indicate that at least one tryptophan, but presumably five residues, are located very close to the retinal chromophore.

Application of the method demonstrated here for bacteriorhodopsin could allow one also to identify amino acids in the neighbourhood of the chromophore of other chromoproteins.

\section{References}

1 Jian, S.-P., Lian, S.-H., Ruan, K.-C., Hui, L.K., Liu, S.-H., Zhan, Z. and Li, Q. (1984) Chem. Phys. Lett. 104, 109-111

2 Graf, F. and Penzhofer, A. (1985) Opt. Quant. Electron. 17, 53-68

3 Oesterhelt, D. (1971) Fed. Proc. 30, 188-188

4 Oesterhelt, D. and Hess, B. (1973) Eur. J. Biochem. 37 , $316-326$

5 Schreckenbach, Th., Walckhoff, B. and Oesterhelt, D. (1978) Biochemistry 17, 5353-5359

6 Kalisky, O., Feitelson, J. and Ottolenghi, M. (1981) Biochemistry 20, 205-209

7 Lanyi, J.K. (1984) in Bioenergetics (Ernster, L., ed.) pp. 315-35, Elsevier, Amsterdam, New York

8 Oesterhelt, D. and Stoeckenius, W. (1974) Methods Enzymol. 31A, 667-678

9 Oesterhelt, D., Schuhmann, L. and Gruber, H. (1974) FEBS Lett. 44, 257-261

10 Polland, H.-J., Franz, M.A., Zinth, W., Kaiser, W., Kölling, E. and Oesterhelt, D. (1984) Biochim. Biophys. Acta 767, 635-639

11 Oesterhelt, D. and Schuhmann, L. (1974) FEBS Lett. 44, 175-178

12 Permyakov, E.A. and Shnyrov, V.L. (1983) Biophys. Chem. $18,145-152$

13 Nuss, M.C., Zinth, W., Kaiser, W., Kölling, E. and Oesterhelt, D. (1985) Chem. Phys. Lett. 117, 1-7

14 Yariv, A. (1975) in Quantum Electronics, John Wiley \& Sons, New York

15 Teale, F.W.J. and Weber, G. (1957) Biochemistry 65, 476-482 
16 Polland, H.J., Franz, M.A., Zinth, W., Kaiser, W., Kölling, E. and Oesterhelt, D. (1986) Biophys. J. 49, 651-662

17 Rehorek, M. and Heyn, M.P. (1979) Biochemistry 18, 4977-4983

18 Sumper, M., Reitmeier, W. and Oesterhelt, D. (1976) Angew. Chemie (Intern. Edn.) 15, 187-194

19 Becher, B. and Cassim, J.Y. (1977) Biophys. J. 19, 285-297

20 Oesterhelt, D. and Hess, B. (1973) Eur. J. Biochem. 37, 316-326

21 Fischer, U. and Oesterhelt, D. (1980) in Abstracts of the VIIIth IUPAC Symposium on Photochemistry, Seefeld (Austria)
22 Becher, B., Tokunaya, F. and Ebrey, T.G. (1978) Bịochemistry $17,2293-2300$

23 Khorana, H.G., Gerber, G.E., Herlihy, W., Gray, C.P., Anderegg, R.J., Nihei, K. and Biemann, K. (1979) Proc. Natl. Acad. Sci. USA 76, 5046-5050

24 Acuna, A.U., González, J., Lillo, M.P. and Oton, J.M. (1984) Photochem. Photobiol. 40, 351-359

25 Trewhella, J., Anderson, S., Fox, R., Gogol, E., Khan, S., Engelman, D. and Zaccai, G. (1983) Biophys. J. 42, 233-241

26 Sherman, W.V. (1982) Photochem. Photobiol. 36, 463-469 